\title{
PEG-b-PCL polymeric nano-micelle inhibits vascular angiogenesis by activating p53-dependent apoptosis in zebrafish
}

This article was published in the following Dove Press journal:

International Journal of Nanomedicine

5 December 2016

Number of times this article has been viewed

\section{Tian Zhou' \\ Qinglei Dong' \\ Yang Shen ${ }^{2}$ \\ Wei Wu' \\ Haide $\mathrm{Wu}^{\prime}$ \\ Xianglin Luo $^{3}$ \\ Xiaoling Liao ${ }^{4}$ \\ Guixue Wang'}

'Key Laboratory for Biorheological Science and Technology of Ministry of Education, State and Local Joint Engineering Laboratory for Vascular Implants, Bioengineering College of Chongqing University, Chongqing, ${ }^{2}$ Institute of Biomedical Engineering, School of Preclinical and Forensic Medicine, Sichuan University, ${ }^{3}$ College of Polymer Science and Engineering, Sichuan University, Chengdu, Sichuan, ${ }^{4}$ Chongqing Key Laboratory of $\mathrm{Nanol}$ Micro Composite Materials and Devices, School of Metallury and Materials Engineering, Chongqing University of Science and Technology, Chongqing, People's Republic of China

Correspondence: Guixue Wang Bioengineering College of Chongqing University, Chongqing University, I74 Shazheng St, Chongqing 400044, People's Republic of China

Tel +862365102508

Fax +862365I0 2507

Email wanggx@cqu.edu.cn
Abstract: Micro/nanoparticles could cause adverse effects on cardiovascular system and increase the risk for cardiovascular disease-related events. Nanoparticles prepared from poly(ethylene glycol) (PEG)- $b$-poly( $\varepsilon$-caprolactone) (PCL), namely PEG- $b$-PCL, a widely studied biodegradable copolymer, are promising carriers for the drug delivery systems. However, it is unknown whether polymeric PEG- $b$-PCL nano-micelles give rise to potential complications of the cardiovascular system. Zebrafish were used as an in vivo model to evaluate the effects of PEG- $b$-PCL nanomicelle on cardiovascular development. The results showed that PEG- $b$-PCL nano-micelle caused embryo mortality as well as embryonic and larval malformations in a dose-dependent manner. To determine PEG- $b$-PCL nano-micelle effects on embryonic angiogenesis, a critical process in zebrafish cardiovascular development, growth of intersegmental vessels (ISVs) and caudal vessels (CVs) in flk1-GFP transgenic zebrafish embryos using fluorescent stereomicroscopy were examined. The expression of fetal liver kinase 1 (flk1), an angiogenic factor, by real-time quantitative polymerase chain reaction (qPCR) and in situ whole-mount hybridization were also analyzed. PEG- $b$-PCL nano-micelle decreased growth of ISVs and CVs, as well as reduced flk1 expression in a concentration-dependent manner. Parallel to the inhibitory effects on angiogenesis, PEG- $b$-PCL nano-micelle exposure upregulated $\mathrm{p} 53$ pro-apoptotic pathway and induced cellular apoptosis in angiogenic regions by qPCR and terminal deoxynucleotidyl transferase dUTP nick end labeling (TUNEL) apoptosis assay. This study further showed that inhibiting p53 activity, either by pharmacological inhibitor or RNA interference, could abrogate the apoptosis and angiogenic defects caused by PEG- $b$-PCL nano-micelles, indicating that PEG- $b$-PCL nano-micelle inhibits angiogenesis by activating p53-mediated apoptosis. This study indicates that polymeric PEG- $b$-PCL nano-micelle could pose potential hazards to cardiovascular development.

Keywords: nanoparticles, PEG- $b$-PCL, cardiovascular diseases, angiogenesis, zebrafish, apoptosis

\section{Introduction}

Nanotechnology develops rapidly and is pioneering the development of drug delivery system (DDS) in biomedical research. Engineered nanoparticles have small size and large surface area, exhibiting distinct properties compared to their counterparts. ${ }^{1}$ Among various types of nanomaterials, nanoparticles prepared from synthetic copolymers have promising applications in DDS due to the biodegradability and biocompatibility, and tunable properties of polymeric materials. ${ }^{2,3}$ Poly(ethylene glycol) (PEG)- $b$ poly ( $\varepsilon$-caprolactone) (PCL), namely PEG- $b$-PCL, represents one of the copolymeric materials that could self-assemble into nano-micelles with core-shell geometry (Figure 1A). Based on this property, increasing efforts have been made to encapsulate 
A
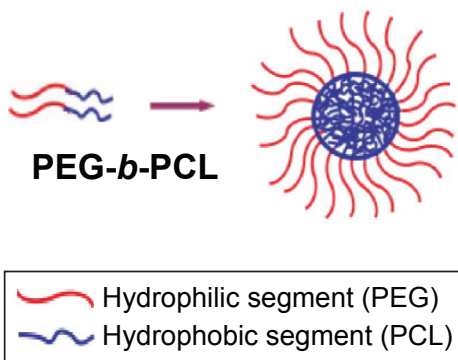

B

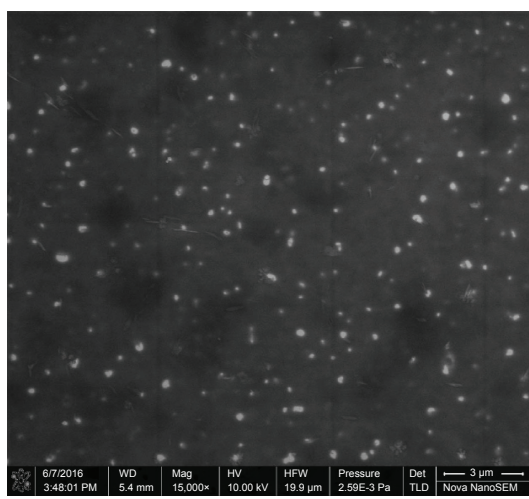

C

Size distribution by intensity

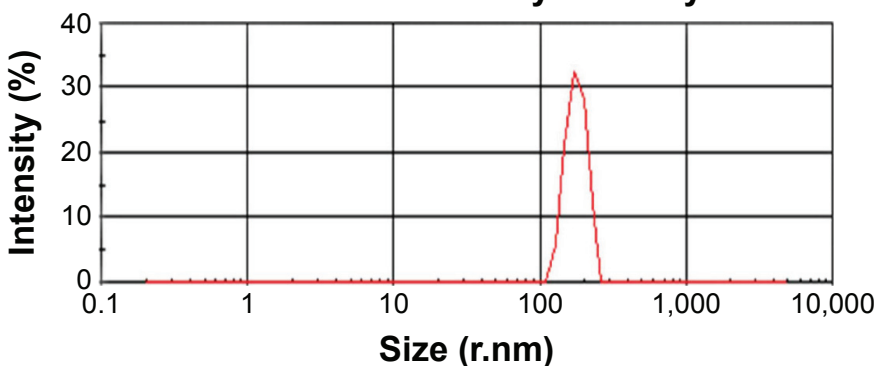

Zeta potential distribution

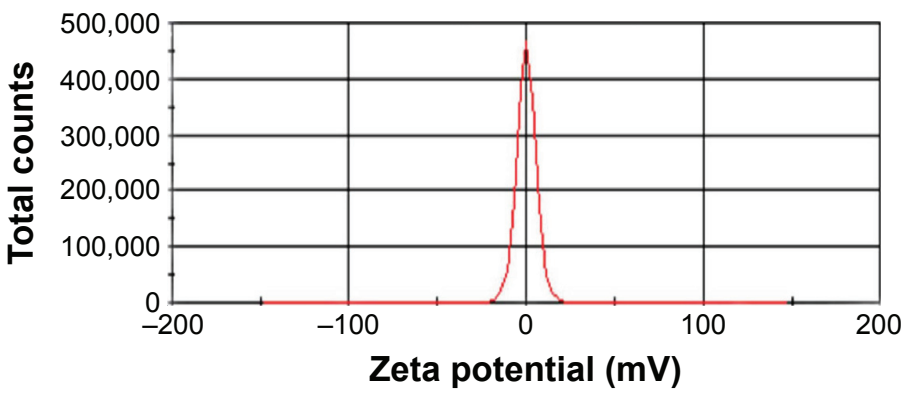

Figure I Characterization of PEG-b-PCL nano-micelles.

Notes: (A) Schematic representation of structure of PEG-b-PCL nano-micelles. (B) Scanning electron microscopy analysis of PEG-b-PCL nano-micelles. (C) Particle size distribution of PEG-b-PCL nano-micelles. (D) Average Zeta potential of PEG-b-PCL nano-micelles.

Abbreviation: PEG-b-PCL, poly(ethylene glycol)-b-poly( $\varepsilon$-caprolactone).

some antitumor drugs with poor water solubility, such as curcumin and doxorubicin into PEG- $b$-PCL nano-micelles to improve therapeutic activities. ${ }^{4,5}$

Despite that PEG and PCL are biocompatible and approved by US FDA in the form of microspheres and implants (containing hydrogels), as well as that PEG- $b$-PCL nano-micelle shows advantages and potentials in DDS, ${ }^{6}$ there are open questions and concerns over the biological behaviors and safety of the nano-sized PEG-PCL, ${ }^{7,8}$ which restricts their further application in clinical therapeutics. The most attractive properties of nanoparticles, such as smaller size and larger surface-to-volume ratio, are also the factors leading to systematic or local toxicity. Since nanoparticles are generally administrated via the intravenous route for therapeutic purposes, the circulatory system is inevitably exposed to the injected nanoparticles. However, it remains unknown whether PEG- $b$-PCL nano-micelle exposure causes any unfavorable responses in the cardiovascular system.

Currently, there are growing concerns over cardiovascular health problems caused by micro/nanoparticles exposure. Epidemiological evidences conducted worldwide have revealed a positive association between adverse cardiovascular events and exposure to airborne particulate matter, especially for fine particles smaller than $2.5 \mu \mathrm{m}\left(\mathrm{PM}_{2.5}\right)$ and ultrafine particles smaller than $100 \mathrm{~nm} .^{9-14}$ The 2010 American Heart
Association scientific statement reached the conclusion that both short-term and chronic exposure to $\mathrm{PM}_{2.5}$ can trigger cardiovascular disease-related events or increase the risk for cardiovascular mortality, while reductions in PM levels are associated with decreases in cardiovascular mortality. ${ }^{10}$ Recent toxicological evaluations of engineered nanoparticles, including nanoparticles of silica ${ }^{15,16}$ and titanium dioxide, ${ }^{17}$ and carbon nanotube, ${ }^{18}$ have demonstrated that exposure to engineered nanoparticles poses risks to cardiovascular functions and causes cardiovascular-related phenotypes in animal models.

Nanoparticles circulate in the bloodstream after intravenous injection while preferentially targeting specific tissues. ${ }^{19,20}$ Bio-distribution study revealed that PEG- $b$-PCL nano-micelles, after intravenous injection, rapidly emerge in the bloodstream within 30 minutes and mostly disappear after 15 hours. ${ }^{21}$ Another type of nano-micelle, which is prepared from PEG-poly-DL-lactide copolymers, showed a similar biological fate, with $25 \%$ of them still circulating in the bloodstream after 24 hours. ${ }^{20}$ Their bio-distribution dynamics in the bloodstream suggest that vascular endothelial cells, the lining cells in blood vessels, are relevant targets of nanoparticles. Indeed, exposure to silica nanoparticle induces cytotoxic damage and decreases cell survival in an umbilical vein cell line, ${ }^{22}$ supporting the fact that nanoparticles trigger some unfavorable responses in the cardiovascular system. 
The current study aims to evaluate the effects of nano-sized PEG- $b$-PCL on cardiovascular development, specifically angiogenesis. Angiogenesis, a process where new vessels originate from preexisting vasculature to form mature blood vessels; it is a critical step in vascular development. Angiogenesis orchestrates a series of activities of endothelial cells, including proliferation, migration, and apoptosis. ${ }^{23,24}$ Zebrafish has been established as a correlative and predictive model for assessing biomaterial toxicity, ${ }^{25,26}$ particularly on cardiovascular development, because the heart is the first organ to form and function during transparent embryo development. ${ }^{27} \mathrm{By}$ employing zebrafish as an vivo model, we first analyzed the overall effects of PEG- $b$-PCL nano-micelles on the morbidity and mortality of zebrafish embryo. We then focused on the in vivo zebrafish embryonic angiogenesis upon exposure to different doses of PEG- $b$-PCL nano-micelles.

To gain insights into the mechanisms regulating nanomicelle effects on angiogenesis, we examined apoptosis within the angiogenic regions and related apoptotic pathways. We found that PEG- $b$-PCL nano-micelle inhibits angiogenesis by inducing apoptosis. Interfering with $\mathrm{p} 53$-mediated apoptosis pathway abrogated apoptosis and restored angiogenesis induced by PEG- $b$-PCL nano-micelles, indicating that PEG- $b$ PCL nano-micelles activate $\mathrm{p} 53$-mediated pro-apoptotic activity to induce cellular apoptosis and inhibit angiogenesis.

\section{Methods}

\section{Preparation of PEG-b-PCL and fluorescein isothiocyanate-labeled PEG-PCL nano-micelles}

$\mathrm{PEG}_{1900}-b-\mathrm{PCL}_{2280}$ block copolymer with $1,900 \mathrm{Da}$ of methoxypoly(ethylene glycol) (mPEG) and 2,280 Da of PCL was synthesized by ring-opening polymerization method according to our previous protocol. ${ }^{28,29}$ Briefly, preweighed mPEG (Alfa Aesar, Tewksbury, MA, USA) and $\varepsilon$-CL monomer (ABCR Company, Karlsruhe, Germany) were mixed in a round-bottomed flask, and the polymerization process was catalyzed by $0.1 \%$ stannous octanoate (Sigma-Aldrich, St Louis, MO, USA) at $130^{\circ} \mathrm{C}$ for 24 hours with nitrogen protection. The obtained copolymer was precipitated in cold methanol. PEG- $b$-PCL self-assembled nano-micelle was prepared by solvent evaporation method as described in our previous studies. ${ }^{28,29}$

Fluorescein isothiocyanate (FITC)-labeled PEG-PCL (PEG- $b$-PCL-FITC) was synthesized as described in other publication. ${ }^{30}$ A total of $0.02 \mathrm{mmol}$ copolymer MPEGPCL was dissolved in $10 \mathrm{~mL}$ dimethyl sulfoxide (DMSO), and then $0.02 \mathrm{mmol}$ FITC (Sigma-Aldrich) was added.
The reaction mixture was heated at $96^{\circ} \mathrm{C}$ for 2 hours in darkness. After cooling, the solution was then dialyzed against distilled water for 3 days and freeze-dried subsequently.

\section{Characterization of PEG- $b$-PCL nano- micelle}

Morphological characteristics, molecular weight, and polydispersity index (PDI) have been described in our previous studies. ${ }^{28,29}$ In this study, we examined morphology of the prepared nanoparticle by scanning electron microscope (Nova400, FEI, Hillsboro, OR, USA) and measured the particle diameter and Zeta potential of PEG- $b$-PCL nanomicelle in distilled water by dynamic light scattering (Nano ZS90, Malvern Instruments, Malvern, UK). To minimize aggregation, the samples were homogeneously dispersed by ultrasound for 10 minutes before use.

\section{Zebrafish maintenance}

Zebrafish were maintained under standard conditions. Adult zebrafish were raised in a recirculated aquarium system with controlled temperature of $28^{\circ} \mathrm{C}$ and humidity of $80 \%$. Photoperiod was set up as 14 hours light/10 hours dark. Zebrafish were fed with brine shrimp daily. Wild-type zebrafish were used for all experiments except for angiogenesis assessment, which was performed with flk1-promoter GFP transgenic zebrafish line (gift from Meng Anming, Tsinghua University, Beijing, People's Republic of China).

\section{Zebrafish exposure to PEG-b-PCL micelle and $\mathrm{p} 53$ inhibitor}

Fertilized eggs were collected within 4 hours postfertilization (hpf). For experiments, embryos were selected under stereomicroscope to ensure they were healthy and were in the same developmental stage. Thirty of the selected embryos were randomly placed in 24-well culture plates with triplicates. At $\sim 7 \mathrm{hpf}$, the embryos were incubated with 60, 120, and $240 \mu \mathrm{g} / \mathrm{mL}$ PEG-b-PCL nano-micelles in baby water until 24-96 hpf. Nano-micelles in the solution were renewed every 24 hours. The embryos in the wells with baby water alone were used as blank control. Embryos were treated with p53 inhibitor, Pifithrin- $\alpha$ ( $4 \mu \mathrm{M}$, Selleck Chemical, Houston, TX, USA) 2 hours prior to nano-micelle treatment. DMSO treatment was also included as DMSO control.

\section{Embryo mortality and malformation}

Dead embryos in every group were counted at 24,48 , and 96 hpf. Embryos with abnormal morphology were also counted. The visible abnormality included pericardial edema, bent tail, and curved spine. Representatives of these 
malformations were imaged by stereomicroscopy (SZX2ILLK, Olympus, Tokyo, Japan).

\section{In vivo zebrafish angiogenesis}

At $24 \mathrm{hpf}$, flk1-GFP transgenic embryos were stripped off the egg sheath and anesthetized with $0.01 \%$ tricaine. Then the embryos were imaged under a fluorescence microscope (MVS10, Olympus) equipped with a standard FITC filter set to monitor angiogenic growth of intersegmental vessels (ISVs) and caudal vessels (CVs). Thirty embryos per replicate were observed to assess the angiogenic growth for every group, followed by quantitative analysis of ISVs length using ImageJ.

\section{Whole-mount in situ hybridization (ISH)}

Probe for flk1 was cloned by polymerase chain reaction (PCR) amplification. Primer sequences for flk1 are

forward 5'-TGCCCAGATTATGGTGATG-3' reverse 5'-CCAAGTCAGCCTATCGTAG-3'.

The PCR products were subcloned into the PMD-20T vector. Digoxigenin (DIG)-labeled antisense RNA probes were synthesized by using a DIG RNA labeling kit (SP6/T7) (Roche, Mannheim, Germany). The procedures for wholemount (ISH) were followed as previously described. ${ }^{31}$

\section{RNA isolation and quantitative RT-PCR}

Thirty embryos from every group were collected for total RNA isolation using RNA simple Total RNA Kit (Tiangen, Beijing, People's Republic of China) according to the manufacturer's instructions. The extracted RNA was measured at 260 and $280 \mathrm{~nm}$ using a spectrophotometer (DU 800 Series, Beckman Coulter, Brea, CA, USA). A total of $500 \mathrm{ng}$ RNA was reverse transcribed to generate cDNA using PrimeScript RT reagent Kit (TaKaRa, Kyoto, Japan) according to the manufacturer's instructions. A total of $10 \mu \mathrm{L}$ reaction system was prepared for real-time quantitative polymerase chain reaction (RT-qPCR) using SYBR Green Master Mix (TaKaRa). The sequences of used primers were fkl forward 5'-GAGAACGGAACCAACAAGATCCAC GAG-3' reverse 5'-CCCTCCAGCAGAACTGACTCCTTAC-3' p53 forward 5'-ACCACTGGGACCAAACGTAG-3' reverse 5'-CAGAGTCGCTTCTTCCTTCG-3', ${ }^{32}$ AIF forward 5'-AAAGTCCGGAAAGAGGGTGT-3' reverse $5^{\prime}$-GCCTGGAGCTCAGCATTAAC-3', ${ }^{33}$ $\beta$-actin forward $5^{\prime}$-CTGTCTTCCCATCCATCG TGGGTC-3' reverse $5^{\prime}$-CTCCATATCATCCCAGTTGGTGACA-3'.
The thermal cycle for RT-qPCR was as follows: $95^{\circ} \mathrm{C}$ for 30 seconds, then 40 cycles of $95^{\circ} \mathrm{C}$ for 10 seconds, $60^{\circ} \mathrm{C}$ for 30 seconds, $72^{\circ} \mathrm{C}$ for 10 seconds, followed by a melting curve analysis to determine the specificity of the reaction. All reactions were run in triplicates using a BioRad real-time PCR system (CFX96 Real Time System). The expression level of target genes was normalized to $\beta$-actin and calculated using the comparative $\mathrm{Ct}$ method.

\section{Whole-mount terminal deoxynucleotidyl transferase dUTP nick end labeling (TUNEL) staining}

Whole-mount TUNEL staining of apoptotic cells in embryos was performed using "In situ cell death detection kit" (Roche) according to manufacturer's instructions. Briefly, 30 embryos at $24 \mathrm{hpf}$ from every group were collected and the chorion was stripped off. The embryos were washed with phosphatebuffered saline (PBS) and fixed with 4\% paraformaldehyde (PFA) at room temperature for 1 hour, followed by incubation with $50 \mu \mathrm{L}$ of TUNEL reaction mixture at $37^{\circ} \mathrm{C}$ for 1 hour in a humid chamber. After PBS wash, the fluorescence was recorded through the FITC channel of a fluorescence microscope, and intensity of TUNEL signal was quantified using ImageJ.

\section{Morpholino injection}

The sequence of morpholino targeting p53 mRNA (p53MO) (Gene Tools, Philomath, OR, USA) is (5'-GCGCCATTGCT TTGCAAGAATTG-3'). The $\mathrm{p} 53 \mathrm{MO}$ was prepared as $1 \mathrm{mM}$ stock solutions in $\mathrm{ddH}_{2} \mathrm{O}$. Around $2.5-5 \mathrm{ng}$ of p53MO, and standard morpholino (stMO) (5'-CCTCTTACCTCAGTTA CAATTTATA-3') as control, were injected into the yolk of embryos at $1-4$ cell stage.

\section{Cell culture, flow cytometry, and immunofluorescence}

Human umbilical vein endothelial cells (HUVECs) were obtained from the American Type Culture Collection (ATCC, Manassas, VA, USA) and cultured in Roswell Park Memorial Institute 1640 medium supplemented with $10 \%$ fetal bovine serum in an incubator with $37^{\circ} \mathrm{C}$ and $5 \% \mathrm{CO}_{2}$.

Apoptosis analysis of HUVECs was performed by flow cytometry according to manufacturer's instructions. Briefly, treated or untreated HUVECs were collected and labeled with Annexin V-APC/7-AAD apoptosis detection kit (KeyGen Biotech, Jiangsu, People's Republic of China), followed by flow cytometry analysis. 
For p53 immunofluorescence, HUVECs were seeded on coverslips in a 24-well plate. After treating HUVECs with 0 (control), 30, and $60 \mu \mathrm{g} / \mathrm{mL}$ PEG- $b$-PCL nano-micelles for 24 hours, the cells were fixed with 4\% PFA for 30 minutes, permeabilized with $0.1 \%$ Triton $\mathrm{X}-100$ for 10 minutes, and blocked with $5 \%$ bovine serum albumin for 1 hour. After blocking, the cells were stained with p53 antibody (rabbit, Santa Cruz Biotechnology Inc, Dallas, TX, USA) overnight at $4{ }^{\circ} \mathrm{C}$, followed by 1 hour incubation with anti-rabbit secondary antibody and 4',6-diamidino-2-phenylindole at room temperature. Samples were imaged using a fluorescence microscopy (Olympus BX81; Olympus).

\section{Statistical analysis}

Data were shown as mean \pm SEM from at least three independent experiments. The differences among groups were determined by one-way analysis of variance followed by Student's $t$-test using Prism 5 (Graphpad, San Diego, CA, USA). Differences were considered statistically significant when $P<0.05$; *denotes $P<0.05$ and **denotes $P<0.01$.

\section{Results}

\section{Characterization of PEG-b-PCL nano- micelles}

$\mathrm{PEG}_{1900}-b$ - $\mathrm{PCL}_{2280}$ copolymeric nano-micelles (PEG- $b$-PCL nano-micelles hereafter) are mostly spherical in morphology, with an average diameter of $173.1 \mathrm{~nm}$, as characterized by scanning electron microscopy (Figure 1B). The particle size distribution and zeta potential distribution of PEG- $b$-PCL nano-micelle were analyzed by the dynamic light scattering. The results showed that the average size of PEG- $b$-PCL micelle is $178.6 \mathrm{~nm}$ (Figure 1C), and the average Zeta potential in distilled water is 0.02 , close to neutral state (Figure 1D). We have previously measured the molecular weight of PCL- $b$ PEG copolymer by ${ }^{1} \mathrm{H}$ nuclear magnetic resonance ( $\left.{ }^{1} \mathrm{H} \mathrm{NMR}\right)$ spectrometry and gel permeation chromatography (GPC). ${ }^{1} \mathrm{H}$ NMR and GPC analysis showed that the number-average molecular weight of PEG- $b$-PCL is 4,100 and 5,000 Da, respectively. ${ }^{28,29}$ The PDI is 1.72 , as measured by GPC with hydrophobic polystyrene as internal standard. ${ }^{29}$

\section{Accumulation of PEG-b-PCL nano-micelle on zebrafish embryo chorion surface}

Zebrafish embryo chorion possesses canals that allow passive diffusion of necessary material for survival. The chorion pore size is $\sim 0.5-0.7 \mu \mathrm{m},{ }^{34}$ which is larger than the prepared PEG- $b$-PCL nano-micelle and it was predicted to cross the chorion. In our study, we used FITC-labeled PEG$b$-PCL nano-micelle to track their localization. PEG- $b$-PCL nano-micelle appeared not to cross the embryo chorion because the conjugated FITC signals predominantly accumulated on the surface of the chorion after removal of the chorion from the embryo (Figure 2). As the concentration of PEG- $b$-PCL nano-micelles increased, more FITC-labeled PEG- $b$-PCL nano-micelles were seen on the embryo chorion. PEG- $b$-PCL did not visibly pass through the chorion to enter the embryos, as reflected by the absence of FITC signals inside embryos.

\section{PEG-b-PCL nano-micelle causes developmental defects in zebrafish embryos}

To evaluate the potential effects of PEG- $b$-PCL nano-micelle, zebrafish embryos at $7 \mathrm{hpf}$ were continuously exposed to PEG- $b$-PCL nano-micelle concentrations ranging from 60 to $480 \mu \mathrm{g} / \mathrm{mL}$. The nano-micelle solutions in every treatment were refreshed every 24 hours. In the group with $480 \mu \mathrm{g} / \mathrm{mL}$ nano-micelle treatment, almost all embryos died after up to $96 \mathrm{hpf}$ (not shown). While 60, 120, and $240 \mu \mathrm{g} / \mathrm{mL}$ nano-micelle caused slight lethality of embryos at 24 and $48 \mathrm{hpf}$, these three sublethal concentrations of nano-micelles dramatically increased embryo mortality at $96 \mathrm{hpf}$ (Figure 3A). The embryo mortality rates at $96 \mathrm{hpf}$ were $\sim 6.6 \%, 11.7 \%$, and $20 \%$ following treatment with 60 , 120 , and $240 \mu \mathrm{g} / \mathrm{mL}$ nano-micelle, indicating a dose and time dependency.

At 72 hpf, almost all embryos hatched. While exposure to 60 and $120 \mu \mathrm{g} / \mathrm{mL}$ PEG- $b$-PCL nano-micelle did not affect embryo hatch $(93.33 \% \pm 1.67 \%$ vs $88.33 \% \pm 1.67 \%$; $93.33 \% \pm 1.67 \%$ vs $88.33 \% \pm 1.67 \%$ ), $240 \mu \mathrm{g} / \mathrm{mL}$ significantly increased embryo hatching rate $(98.33 \% \pm 1.67 \%$ vs $88.33 \% \pm 1.67 \%$ ) (Figure S1). Interestingly, we found that, when stripping off the chorion with forceps, the chorionic membrane became more fragile after embryo exposure to $240 \mu \mathrm{g} / \mathrm{mL}$ PEG- $b$-PCL nano-micelle, while the embryo chorions were quite elastic in the control group and the other two exposed groups (not shown). These suggest a possibility that PEG- $b$-PCL nano-micelle attaching on the chorion surface alters the physical properties of chorionic membrane, which could possibly increase hatch of zebrafish embryo.

Toxicity of nanoparticles is frequently associated with malformations in zebrafish embryo and larva. ${ }^{16,35,36} \mathrm{We}$ examined whether PEG- $b$-PCL nano-micelle induced malformations in zebrafish. As shown in Figure 3B, at $48 \mathrm{hpf}$, embryos developed normally upon treatment with 

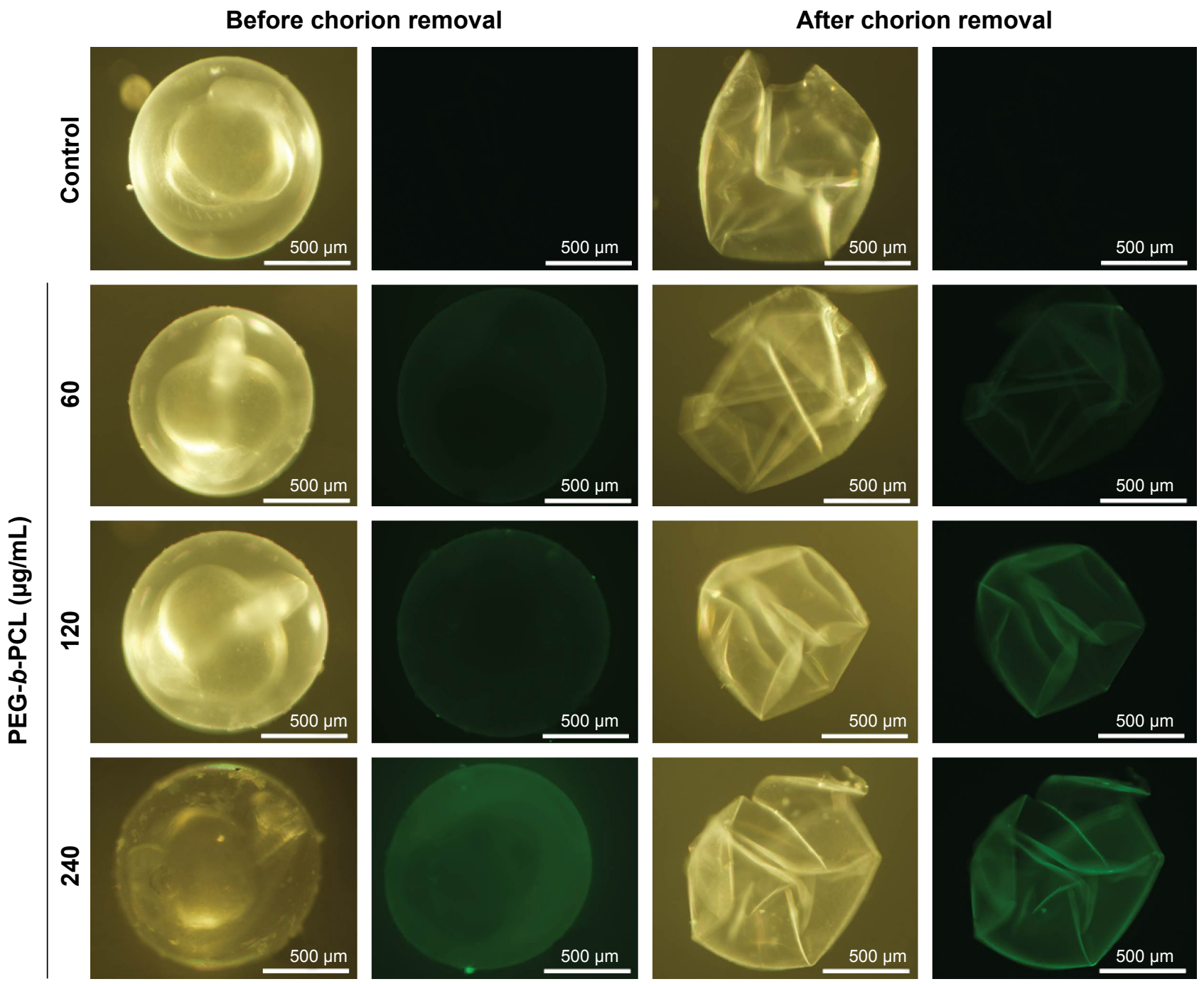

Figure 2 Embryo chorion absorption of PEG-b-PCL nano-micelles.

Notes: Embryos were exposed to 0, 60, I20, and $240 \mu \mathrm{g} / \mathrm{mL}$ FITC-labeled PEG-b-PCL nano-micelles. At 24 hpf, embryo chorions were manually removed. Bright-field and fluorescent images of embryos before and after chorion removal are shown.

Abbreviations: hpf, hours postfertilization; FITC, fluorescein isothiocyanate; PEG-b-PCL, poly(ethylene glycol)-b-poly( $\varepsilon$-caprolactone).

60 and $120 \mu \mathrm{g} / \mathrm{mL}$ nano-micelle, while embryos exposed to $240 \mu \mathrm{g} / \mathrm{mL}$ developed pericardial edema and deformed yolk sac, as well as bent tail. As embryos developed to $72 \mathrm{hpf}, 120 \mu \mathrm{g} / \mathrm{mL}$ nano-micelle also exerted toxicity, causing pericardial edema, while $240 \mu \mathrm{g} / \mathrm{mL}$ exposure caused pericardial edema, deformed yolk sac, and bent tail. These malformations looked more severe at $96 \mathrm{hpf}$ embryos, which also developed bent tail in $120 \mu \mathrm{g} / \mathrm{mL}$ exposure group and became dead in $240 \mu \mathrm{g} / \mathrm{mL}$ group. We quantified the percentage of abnormal embryos caused by nano-micelle toxicity (Figure 3C) and found that 120 and $240 \mu \mathrm{g} / \mathrm{mL}$ nano-micelle significantly increased the incidence of embryo malformations, while low dose-treated embryos showed no statistical difference with control group (Figure 3C). These data indicate that exposure to PEG- $b$-PCL nano-micelle caused developmental defects in zebrafish embryos in a dose- and time-dependent manner.

\section{PEG-b-PCL nano-micelle inhibits angiogenesis in zebrafish embryo}

We then analyzed the effects of PEG- $b$-PCL nano-micelle on blood vessel development of zebrafish embryos. Using flk1GFP transgenic zebrafish as in vivo angiogenesis model, we could readily monitor angiogenic development after embryo exposure to nano-micelle. Similar to control group, embryos exposed to $60 \mu \mathrm{g} / \mathrm{mL}$ of nano-micelle developed normal ISVs and CVs at $24 \mathrm{hpf}$. However, higher doses of nano-micelle treatment resulted in a dramatic impairment of vascular growth. ISV number and length were significantly decreased after embryo exposure to 120 and $240 \mu \mathrm{g} / \mathrm{mL}$ nano-micelle 
A

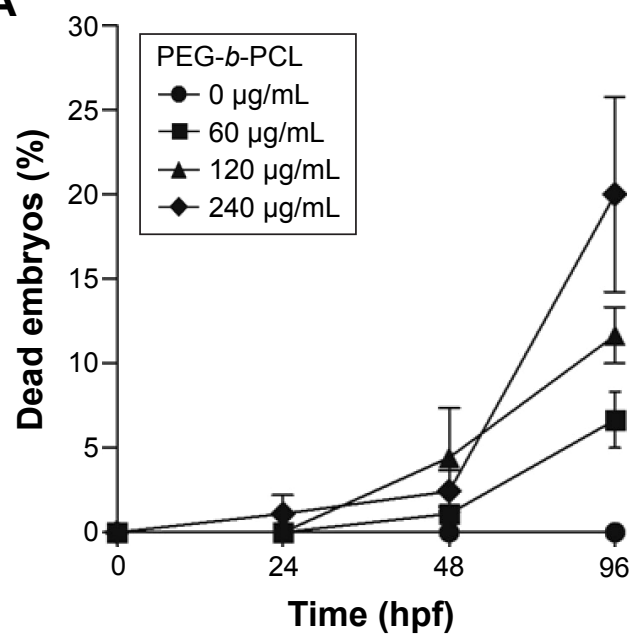

C

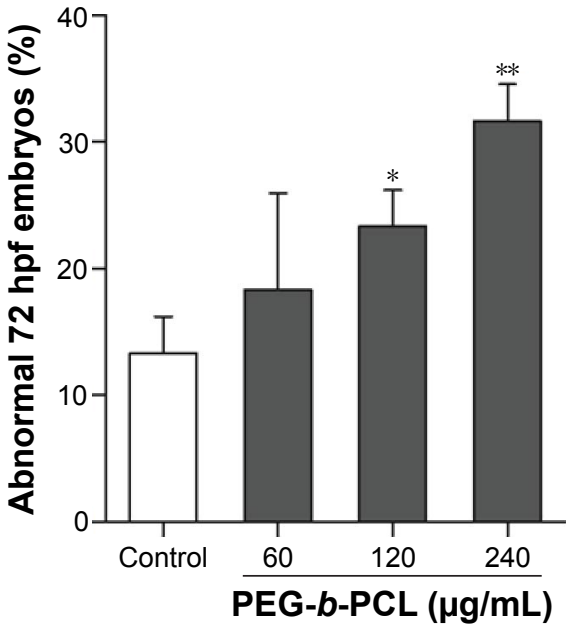

B

PEG-b-PCL $(\mu \mathrm{g} / \mathrm{mL})$
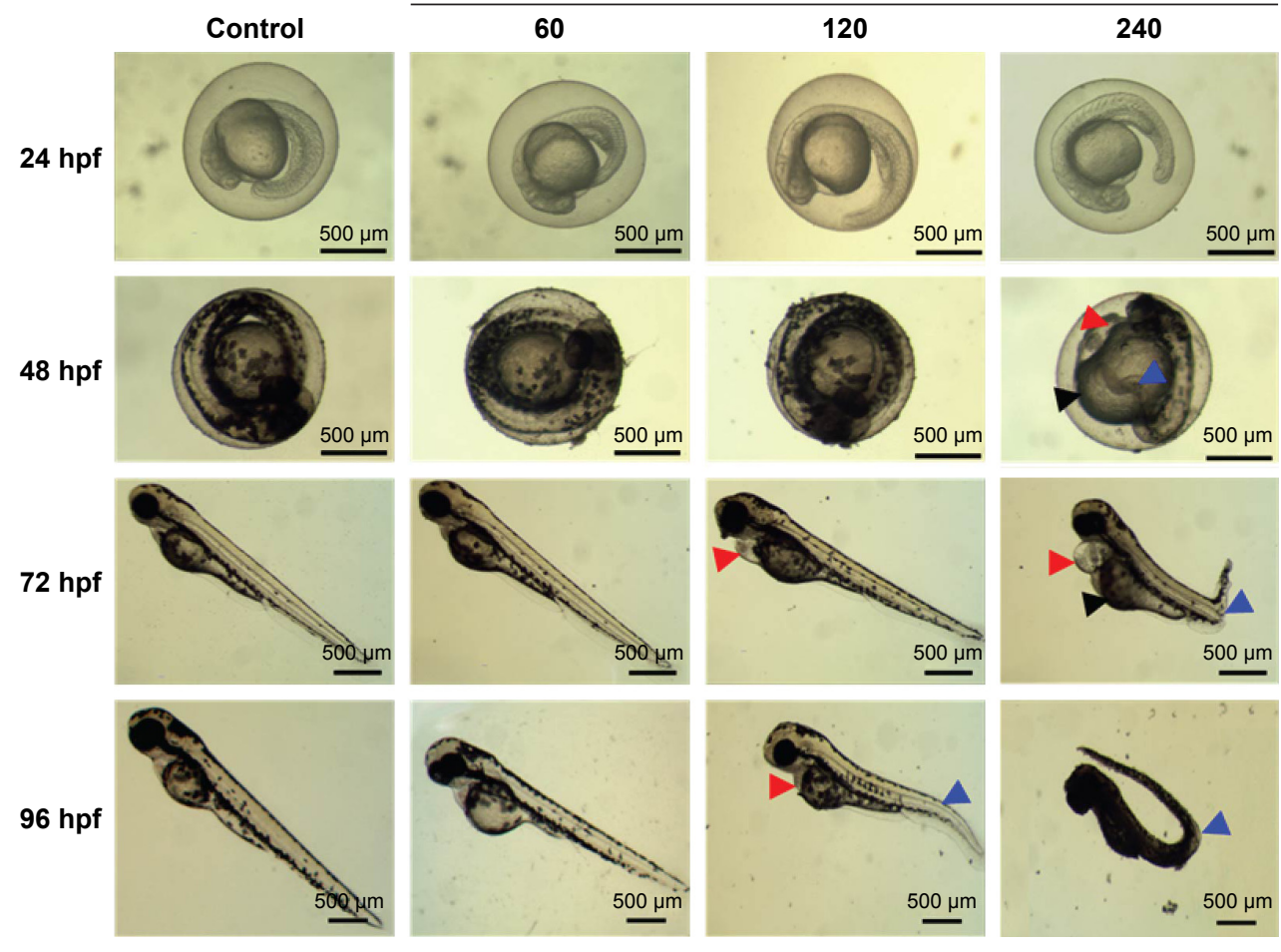

Figure 3 Effects of PEG-b-PCL on zebrafish embryos and larva.

Notes: (A) The mortality rates of embryos exposed to 0, 60, 120, and $240 \mu \mathrm{g} / \mathrm{mL}$ PEG-b-PCL nano-micelles. (B) Representative images showing the morphological changes of embryos exposed to different doses of nano-micelle at indicated stages. Red arrowheads indicate pericardial edema, black arrowheads indicate yolk sac deformation, and blue arrowheads indicate tail curve. (C) Quantification of $72 \mathrm{hpf}$ embryos showing malformations, including pericardial edema, yolk sac deformation, and tail curve. Data are expressed as mean \pm SEM from three independent experiments $(n=180)$, $* P<0.05, * * p<0.01$.

Abbreviations: hpf, hours postfertilization; PEG-b-PCL, poly(ethylene glycol)-b-poly( $\varepsilon$-caprolactone); SEM, standard error of mean.

(Figure 4A and B). The $240 \mu \mathrm{g} / \mathrm{mL}$ nano-micelle-induced vascular impairment also includes defect in CVs, where the vascular cavity was reproducibly seen (Figure 4A), indicating that nano-micelle has apparent toxicity on embryonic vessel development.

To confirm the angiogenic defects caused by nanomicelle exposure, we examined vascular development by whole-mount ISH of vascular endothelial marker, flk1. Vascular defects were observed after exposure to 120 and $240 \mu \mathrm{g} / \mathrm{mL}$ nano-micelles as revealed by reduced or absent $f l k 1$ mRNA expression in ISVs (Figure 4C). The $f k 1$ mRNA expression was quantified by qPCR assessment. Consistent with ISH of $f k 1$, there was a robust decrease after $120 \mu \mathrm{g} / \mathrm{mL}$ $(P<0.05)$ and $240 \mu \mathrm{g} / \mathrm{mL}(P<0.01)$ nano-micelle exposure, 
A
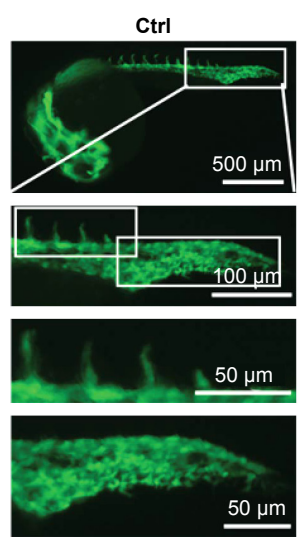

$50 \mu \mathrm{m}$

C
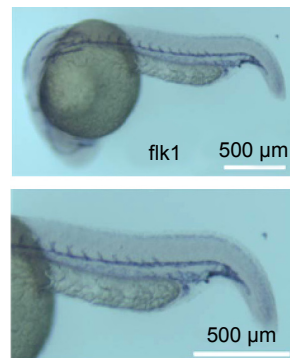

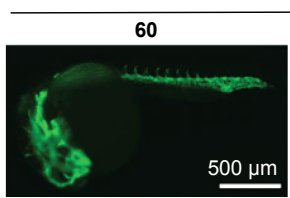

PEG-b-PCL $(\mu \mathrm{g} / \mathrm{mL})$
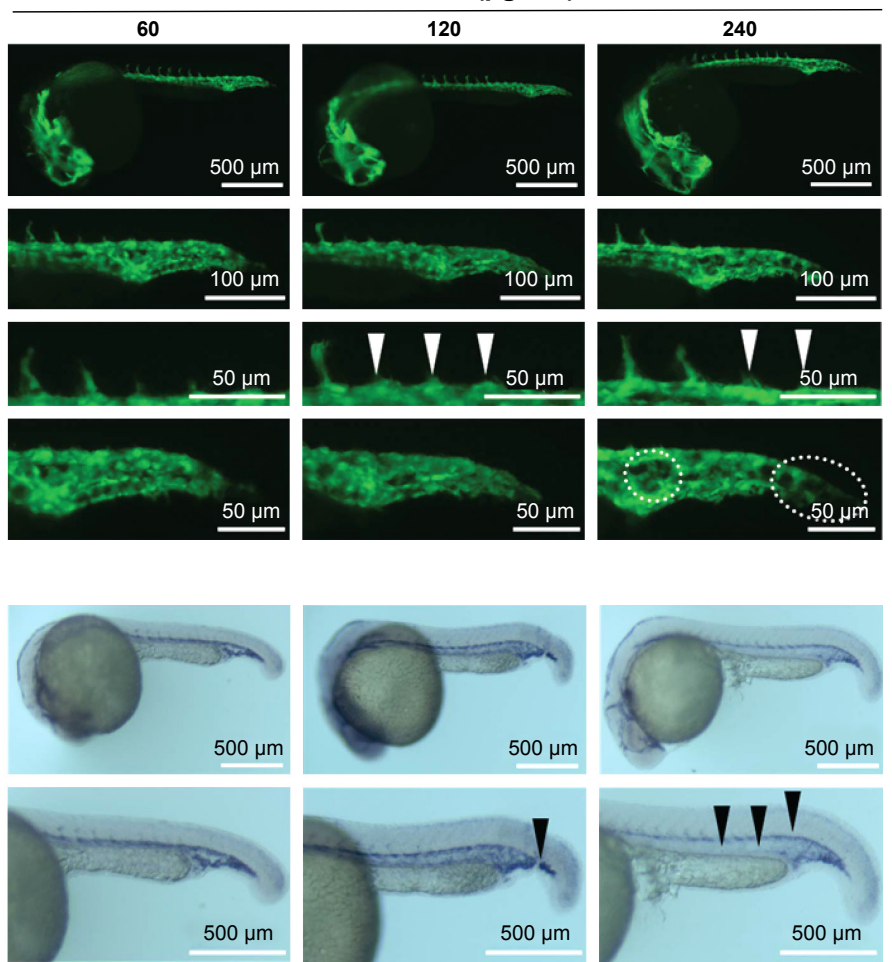

B

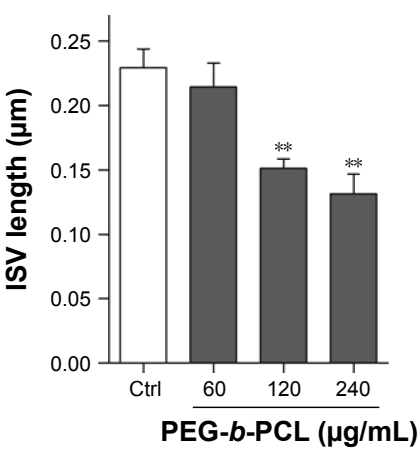

D

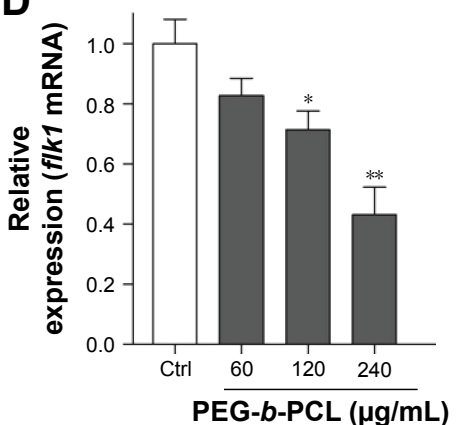

Figure 4 PEG-b-PCL nano-micelle inhibits angiogenesis in zebrafish embryo.

Notes: (A) Fluorescent images of flkl-GFP positive vascular sprouting in 24 hpf unexposed embryos (Ctrl) and embryos exposed to 60 , I20, and $240 \mu \mathrm{gg} / \mathrm{mL}$ PEG-b-PCL nano-micelle. The top images are zoomed in the second top images, which are further zoomed in the bottom two images. White arrowheads indicate the absence of ISV and the dashed ovals indicate cavity in caudal vessels. (B) Quantification of ISV length in 24 hpf control embryos and embryos exposed to the indicated doses of PEG-b-PCL nano-micelles. (C) In situ hybridization analysis of flk I expression in 24 hpf control embryos and embryos exposed to the indicated doses of PEG-b-PCL nano-micelles. The bottom images show the zoomed ISVs and caudal vessels. The black arrowheads indicate the absence of flkI signal. (D) q-PCR analysis of flkI mRNA expression in 24 hpf control and PEG-b-PCL treated embryos. Data are expressed as mean \pm SEM from three biological samples. $* P<0.05, * * P<0.01$.

Abbreviations: Ctrl, control; hpf, hours postfertilization; ISV, intersegmental vessel; PEG-b-PCL, poly(ethylene glycol)-b-poly( $\varepsilon$-caprolactone); qPCR, quantitative polymerase chain reaction; SEM, standard error of mean.

while no significant change in $f k 1$ mRNA level was observed after $60 \mu \mathrm{g} / \mathrm{mL}$ nano-micelle treatment (Figure 4D). All together, these data strengthen the concentration-dependent nanotoxicity of PEG- $b$-PCL nano-micelle on vascular development.

\section{PEG-b-PCL nano-micelle activates p53- dependent apoptosis}

To gain insights into the mechanisms underlying angiogenic defects caused by PEG- $b$-PCL nano-micelles, we examined apoptosis in vivo by whole-mount TUNEL assay. After $240 \mu \mathrm{g} / \mathrm{mL}$ nano-micelle exposure, apoptotic cells, as indicated by TUNEL-positive cells at ISVs and CVs, were markedly increased when compared with control group (Figure 5A and B). As expected, $60 \mu \mathrm{g} / \mathrm{mL}$ treatment did not increase apoptosis significantly (Figure 5A and B). Interestingly, apoptotic cells within those vascular regions sensitive to relatively high dose exposures remained comparable between $120 \mu \mathrm{g} / \mathrm{mL}$ exposure and control group (Figure 5B), suggesting other cellular processes rather than apoptosis were involved in angiogenesis inhibition by nano-micelle. To ascertain the apoptosis induction, we measured expression levels of two apoptosis-related genes, $p 53$ and apoptosis inducing factor $(A I F)$. In accordance with TUNEL-positive apoptotic cells, mRNA levels of both genes were increased after treatment of $240 \mu \mathrm{g} / \mathrm{mL}$ nano-micelle, rather than 60 and $120 \mu \mathrm{g} / \mathrm{mL}$, indicating high dose of nano-micelle activates apoptosis during impaired vascular sprouting.

We confirmed that PEG- $b$-PCL nano-micelles induced apoptosis in HUVECs, a widely used cell line for studying vascular endothelial responses in vitro. Flow cytometry results showed that 30 and $60 \mu \mathrm{g} / \mathrm{mL}$ PEG- $b$-PCL nanomicelles could increase the populations of apoptotic cells to some extent (Figure S2A). Additionally, p53 immunostaining showed that p53 expression appears stronger in HUVECs after 30 and $60 \mu \mathrm{g} / \mathrm{mL}$ PEG- $b$-PCL nano-micelles treatment 
A

PEG-b-PCL $(\mu \mathrm{g} / \mathrm{mL})$
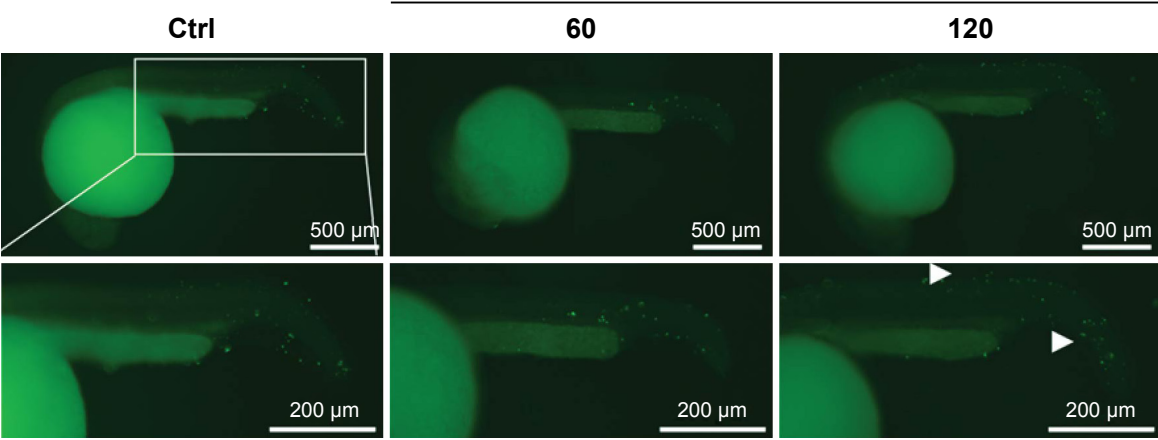

240

B

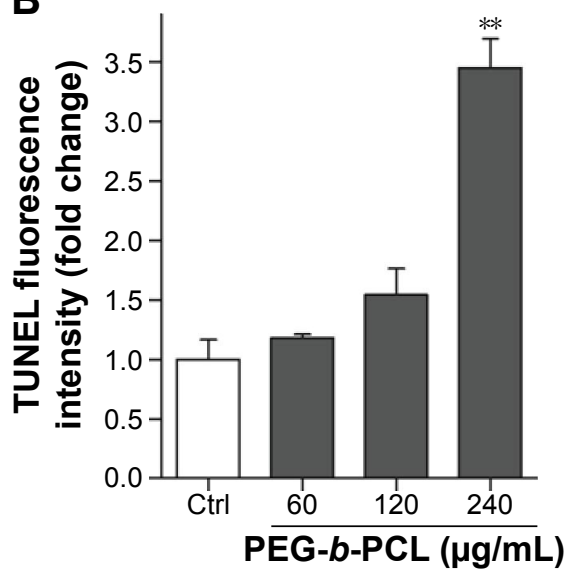

C

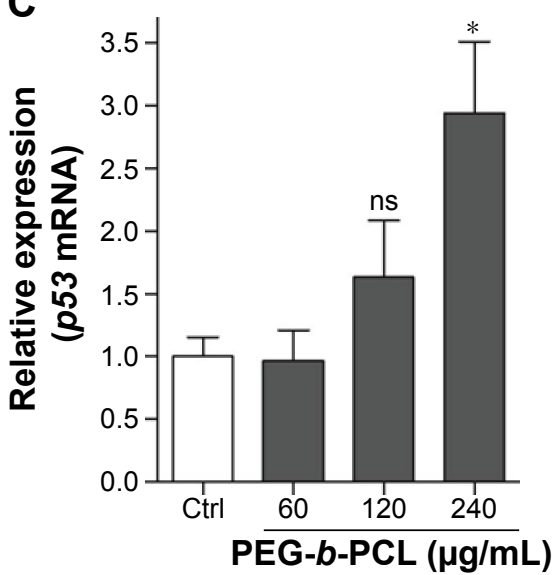

D

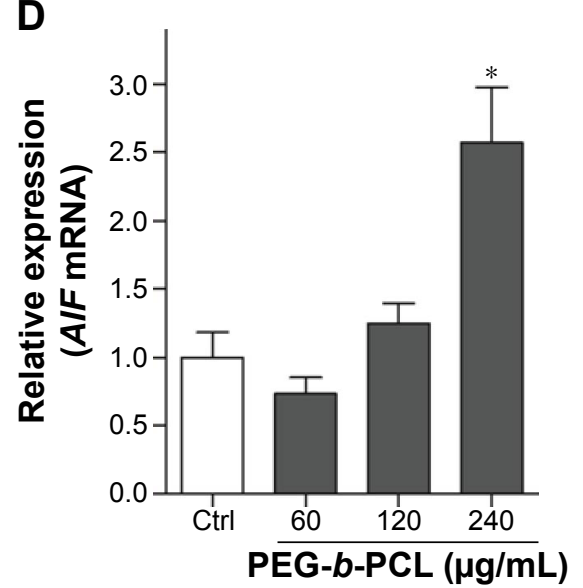

Figure 5 PEG-b-PCL nano-micelle induces apoptosis in zebrafish embryos.

Notes: (A) TUNEL analysis of apoptosis in control and PEG-b-PCL nano-micelle treated 24 hpf embryos. The bottom images showed the zoomed vessels. The white arrowheads indicate apoptotic cells. (B) Mean fluorescence intensity measurement of apoptosis shown in (A). (C, D) qPCR analysis of p53 and AIF mRNA expression in 24 hpf control and PEG-b-PCL exposed embryos. Data are expressed as mean \pm SEM from three biological samples. $* P<0.05$, $* * P<0.01$.

Abbreviations: Ctrl, control; hpf, hours postfertilization; PEG-b-PCL, poly(ethylene glycol)-b-poly( $\varepsilon$-caprolactone); qPCR, quantitative polymerase chain reaction; TUNEL, terminal deoxynucleotidyl transferase dUTP nick end labeling; SEM, standard error of mean; ns, not significant.

(Figure S2B). Thus, results from these two in vitro assays corroborated the apoptosis phenotype from the whole fish.

Given p53 is a key player in apoptosis, and PEG- $b$-PCL nano-micelles induced transcriptional upregulation of $p 53$ and increased apoptosis, we consider the possibility that PEG- $b$-PCL nano-micelle triggers apoptosis dependent on p53 activity. If so, inhibiting p53 activity could restore the developmental defects caused by PEG- $b$-PCL nanomicelle. To this end, we inhibited the activity of p53 either by pretreating $7 \mathrm{hpf}$ embryos with specific inhibitor of $\mathrm{p} 53$, Pifithrin- $\alpha$, or by interfering p53 mRNA activity of $1-4$ cell stage embryos with p53 morpholino (p53MO). DMSO and standard MO (stMO) were used as control for Pifithrin- $\alpha$ and $\mathrm{p} 53 \mathrm{MO}$, respectively. DMSO and stMO did not cause obvious effects on developmental processes, including apoptosis and angiogenesis (not shown). As shown in Figure 6A, in those unexposed embryos, Pifithrin- $\alpha$ pretreatment and p53MO preinjection showed no effects on apoptosis within
ISVs and CVs regions. However, the increased number of TUNEL-positive cells within ISVs and CVs in nanomicelle exposure group was significantly decreased when the embryos were pretreated with Pifithrin- $\alpha$ or preinjected with p53MO. Notably, the apoptosis even decreased to the basal level, as seen in embryos without nano-micelle exposure (Figure 6B). These data indicate that PEG- $b$-PCL nano-micelles induce vascular apoptosis dependent on p53 pro-apoptotic activity.

\section{p53 inhibition abrogates the angiogenic defects caused by PEG-b-PCL nano- micelle}

To further determine the role of $\mathrm{p} 53$ in developmental defects caused by PEG- $b$-PCL nano-micelle, we monitored vascular angiogenesis of 24 hpf embryos pretreated with Pifithrin- $\alpha$ or preinjected with $\mathrm{p} 53 \mathrm{MO}$ followed by PEG- $b$-PCL nanomicelle exposure. As observed by fluorescent microscopy 
A

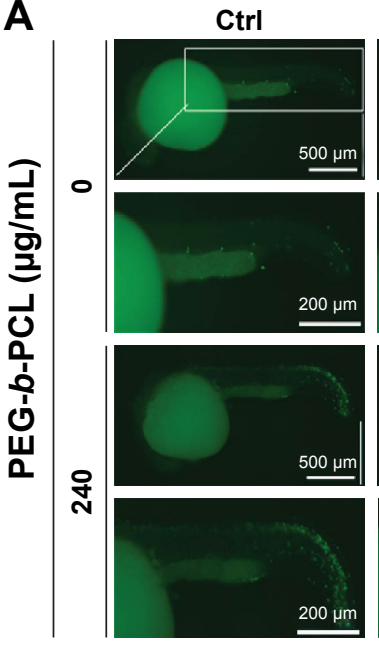

Pifithrin- $\alpha$

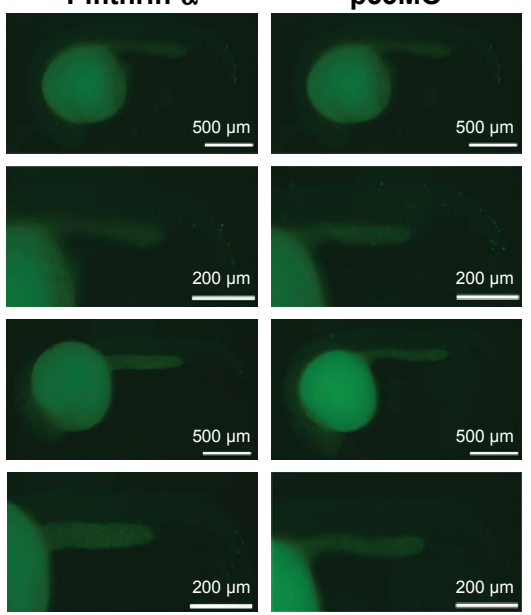

B

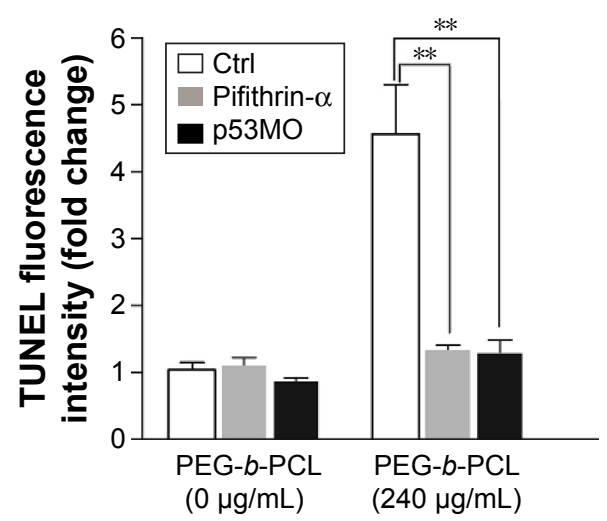

Figure 6 Inhibiting p53 activity abrogates apoptosis caused by PEG-b-PCL nano-micelle.

Notes: (A) TUNEL analysis of apoptosis in control, Pifithrin- $\alpha$ (p53 inhibitor) pretreated and p53 morpholino (p53MO) preinjected embryos with or without $240 \mu \mathrm{gg} / \mathrm{mL}$ PEG$b$-PCL exposure. (B) Mean fluorescence intensity measurement of apoptosis shown in (A). Data are expressed as mean $\pm \mathrm{SEM}$ from three biological samples. $* * P<0.0 \mathrm{I}$. Abbreviations: Ctrl, control; MO, morpholino; PEG-b-PCL, poly(ethylene glycol)-b-poly( $\varepsilon$-caprolactone); TUNEL, terminal deoxynucleotidyl transferase dUTP nick end labeling; SEM, standard error of mean.

and ISH as well as RT-qPCR, Pifithrin- $\alpha$ pretreatment or p53MO preinjection did not cause vascular growth defects in unexposed embryos, indicating Pifithrin- $\alpha$ and p53MO have no effects on normal developmental processes. Nanomicelle exposure impaired angiogenesis at $24 \mathrm{hpf}$ embryo (Figures 4A and 7A). However, the impaired angiogenesis in nano-micelle exposure group was rescued when $p 53$ activity was inhibited either by pretreating embryos with Pifithrin- $\alpha$ or by preinjecting with $\mathrm{p} 53 \mathrm{MO}$ (Figure 7B-D). Thus, inhibition of p53 activity abrogates angiogenic defects caused by nano-micelles.

\section{Discussion}

Amphiphilic copolymer-constructed nanoparticles hold promises in biomedical field, for example, in the development of DDS. Prior to their application, it is essential to fully understand their biocompatibility. In recent years, it has been revealed that exposure to engineered nanoparticles is associated with increased risks of cardiovascular health problems, which gained attention from scientific community and the public. In this study, we provided evidences to show that polymeric PEG- $b$-PCL nano-micelles could increase mortality and morbidity, as well as impair cardiovascular system. Using zebrafish as a model, we showed that PEG$b$-PCL nano-micelles exert dose-dependent restriction on zebrafish development, causing embryonic lethality and malformations, including pericardial edema and bent tail. Specifically, we then focused on the development of the cardiovascular system and found that PEG- $b$-PCL nanomicelles exposure compromises embryonic angiogenesis, one of the primary processes involved in cardiovascular development in zebrafish. Mechanistically, we showed that PEG- $b$-PCL upregulates $\mathrm{p} 53$ pathway and triggers apoptosis. Suppression of $\mathrm{p} 53$ activity abrogates PEG- $b$-PCL effects on vascular angiogenesis, demonstrating that $\mathrm{p} 53$-mediated apoptosis contributes to the inhibitory effects of PEG- $b$-PCL nano-micelles on vascular angiogenesis.

It is critical to analyze the uptake of PEG- $b$-PCL nanomicelle by the zebrafish embryo chorion. The chorion has canals or pores that allow transport of oxygen and nutrient and other materials to embryo via passive diffusion. Embryo chorion is thought to protect against nanoparticle entry. ${ }^{34,37}$ Though the size of chorion canals is $\sim 0.5-0.7 \mu \mathrm{m}$ in diameter, ${ }^{34}$ larger than the common size of nanoparticles, it remains controversial whether nanoparticles could penetrate the chorion. For example, $20 \mathrm{~nm}$ nano-silver is found to cross chorion, ${ }^{34}$ while 10 or $35 \mathrm{~nm}$ nano-silver failed to penetrate the chorion. ${ }^{35}$ Similarly, 50 and $200 \mathrm{~nm}$ nano-silica do not visibly cross the chorion..$^{38}$ The apparent discrepancy could be likely due to the nanoparticle agglomeration or other unknown mechanisms. The adsorbed nanoparticles on the chorion surface might block the pores and affect nutrient transport. In the present study, using FITC-labeled PEG- $b$-PCL nano-micelles to track their localization, we found the predominant accumulation of FITC signal on the surface of the chorion upon its removal from the embryo 
A

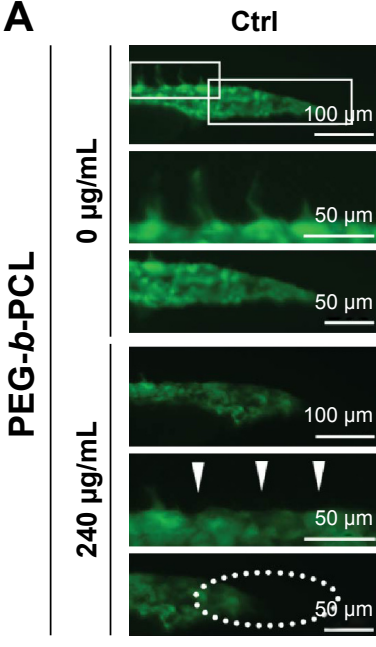

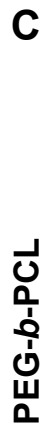

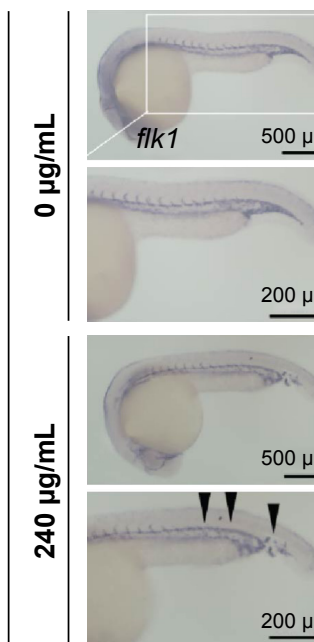

Pifithrin- $\alpha$
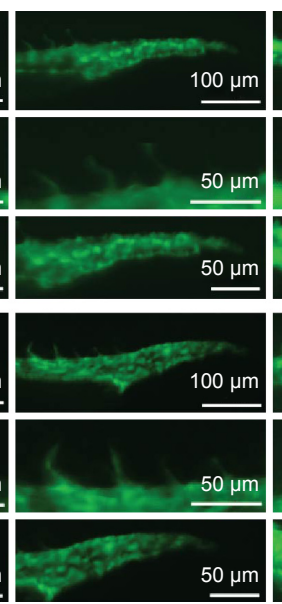

$\mu \mathrm{m}$

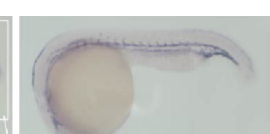

$5 \underline{\underline{5 m}}$

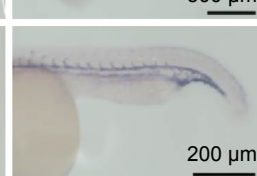

$200 \mu \mathrm{m}$

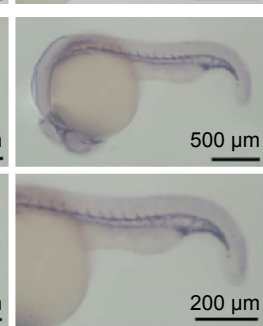

p53MO
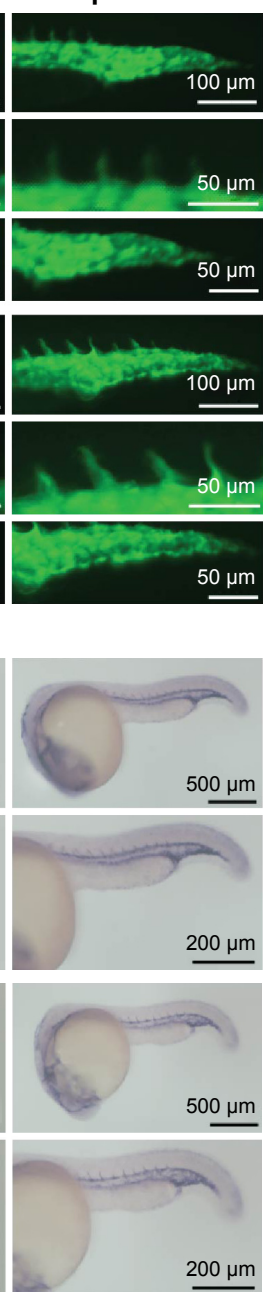

B

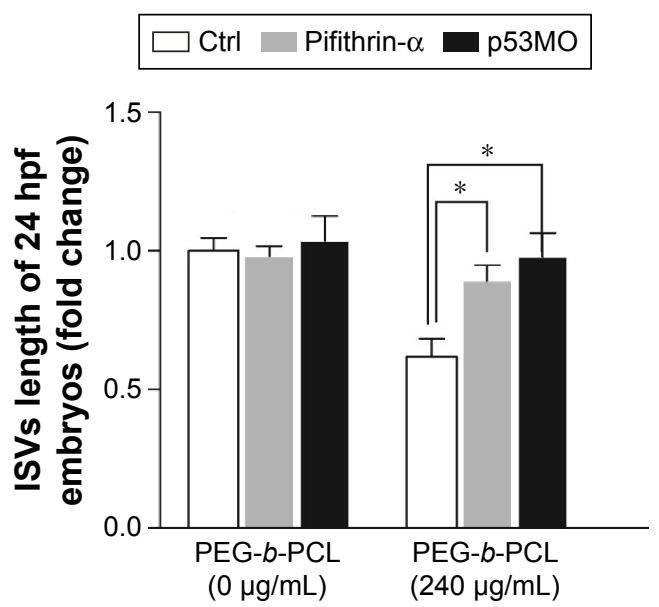

D

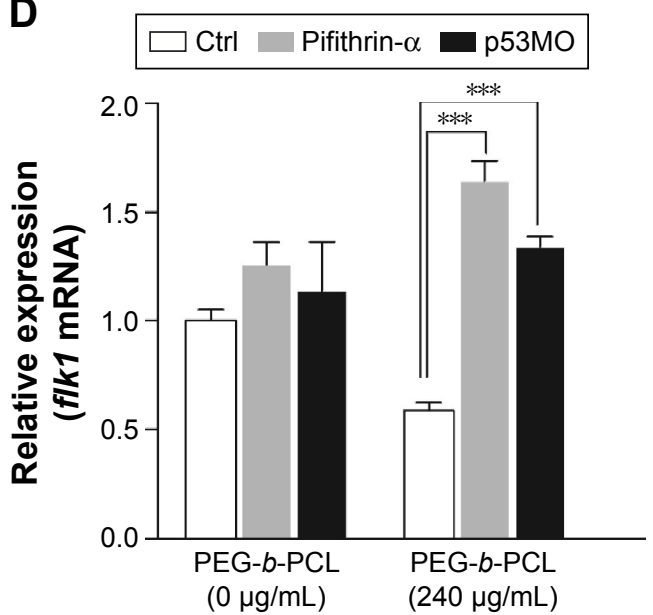

Figure 7 p53 inhibition abrogates the angiogenic defects caused by PEG-b-PCL nano-micelle.

Notes: (A) Representative images of flkl-GFP-positive vascular sprouting in $24 \mathrm{hpf}$ control, Pifithrin- $\alpha$ pretreated and p53MO preinjected embryos with or without $240 \mu \mathrm{g} / \mathrm{mL}$ PEG-b-PCL exposure. White arrowheads indicate the absence of ISV and the dashed oval indicates the cavity in caudal vessels. (B) Quantification of ISV length as shown in (A). (C) In situ hybridization analysis of flk l expression in $24 \mathrm{hpf}$ control, Pifithrin- $\alpha$ pretreated and p53MO preinjected embryos with or without $240 \mu \mathrm{g} / \mathrm{mL}$ PEG-b-PCL exposure. Black arrowheads indicate the absence of flk $l$ expression. (D) qPCR analysis of flk $/ \mathrm{mRNA}$ expression in 24 hpf control, Pifithrin- $\alpha$ pretreated and P53MO preinjected embryos with or without $240 \mu \mathrm{g} / \mathrm{mL}$ PEG-b-PCL exposure. Data are expressed as mean \pm SEM from three biological samples. $* P<0.05$, $* * * P<0.00 \mathrm{I}$. Abbreviations: Ctrl, control; hpf, hours postfertilization; ISV, intersegmental vessel; MO, morpholino; PEG-b-PCL, poly(ethylene glycol)-b-poly( $\varepsilon$-caprolactone); qPCR, quantitative polymerase chain reaction; SEM, standard error of mean.

(Figure 2). However, we did not take a closer look at the detailed distribution of PEG- $b$-PCL nano-micelles in specific organs of the exposed zebrafish embryos. Thus, it is currently unknown whether the chorion protects against uptake of PEG- $b$-PCL and future work will be needed to determine their systemic distribution after embryo or larval exposure.

Angiogenesis, originating from the preexisting blood vessels, plays important physiological roles in embryonic development and tissue repair. ${ }^{39}$ Impaired blood vessel formation results in inadequate blood supply and triggers cardiovascular events. Angiogenesis is also implicated in diverse pathological settings, like tumor growth. ${ }^{39}$ Excessive microvessel formation within a tumor sustains its growth by providing oxygen and nutrients. Among the growth factors that regulate angiogenesis, the members of vascular endothelial growth factor (VEGF) family proteins are potent angiogenic factors for physiological and pathological angiogenesis. ${ }^{40}$ VEGF receptor 2 (also known as flk1) is an endothelial-specific receptor for VEGF, and mediates diverse biological effects of VEGF on endothelial cells, including survival, proliferation, and migration. ${ }^{40}$ During zebrafish embryogenesis, flk1 is highly expressed in vascular endothelial cells during the formation of blood vessel. Consistent with the importance of VEGF/flk1 signaling in embryonic angiogenesis, zebrafish embryo lacking flk1 disruption impairs the formation of blood vessel generated by 
sprouting angiogenesis. ${ }^{41,42}$ In the present study, by measuring the growth of ISVs and CVs in 24 hpf zebrafish embryos, we found that PEG- $b$-PCL nano-micelles exposure inhibits embryonic angiogenesis (Figure 4A and B). Consistently, flk1 expression is downregulated after exposure to PEG- $b$ PCL nano-micelle (Figure 4C and D).

As endothelial cell apoptosis is an inhibitory process of angiogenesis, we suspect that increased apoptosis is the cause for angiogenesis defects by nano-micelle. Excessive apoptosis, differing from proliferation, could limit angiogenesis and cause vessel regression. ${ }^{24}$ Growth factors such as VEGF that stimulate cell proliferation and migration are also antiapoptotic. ${ }^{24,43}$ PEG- $b$-PCL nano-micelle induces apoptosis, as revealed by increased TUNEL-positive cells in angiogenic sites (Figure 5A and B) and upregulated expression of apoptotic genes, p53 and AIF (Figure 5C and D). We then revealed that PEG- $b$-PCL nano-micelles induce apoptosis in the vasculature via a p53-dependent manner. Using pharmacological inhibitor of $p 53$ or RNAi knockdown against $\mathrm{p} 53$, we found that these two approaches suppressed p53-mediated apoptosis and also restored embryonic angiogenesis, supporting the notion that PEG- $b$-PCL nano-micelles inhibit angiogenesis by activating apoptosis. It has been well demonstrated in many types of nano-materials that increased apoptosis is one of the common hazards from nanomaterials. ${ }^{16,44}$ It appears that p53-mediated apoptosis is the primary cause for angiogenesis inhibition after PCL- $b$-PEG nano-micelle exposure, because inhibition of p53 is sufficient to abrogate angiogenic defects caused by PCL- $b$-PEG nanomicelle. However, we could not exclude the involvement of other apoptotic pathways in this process.

\section{Conclusion}

In this study, we found that PEG- $b$-PCL nano-micelle impairs cardiovascular development in zebrafish. PEG- $b$-PCL nano-micelle inhibits zebrafish embryonic angiogenesis by upregulating $p 53$ expression and inducing apoptosis. Inhibiting p53 abrogates these adverse effects, indicating that p53-mediated apoptosis contributes to PEG- $b$-PCL nano-micelles effects on angiogenesis and cardiovascular development.

\section{Acknowledgments}

We would like to thank Yiming Zheng from the Department of Biomedical Sciences in Florida State University for help with manuscript preparation. We thank Dr Xiang Xie from the College of Bioengineering in Chongqing University for technical assistance. This study was supported by grants from the
National Natural Science Foundation of China (111572064, 11332003), by the cooperative project of Chongqing Key Laboratory of Nano/Micro Composite Materials and Devices (CQKL-1502) and the National Key Technology R \& D Program of China (2012BAI18B02). The authors also thank the support of experimental instruments from the National "111 Plan" Base (B06023) and the Public Experiment Center of State Bioindustrial Base, Chongqing, People's Republic of China.

\section{Disclosure}

The authors report no conflicts of interest in this work.

\section{References}

1. Johnston H, Pojana G, Zuin S, et al. Engineered nanomaterial risk. Lessons learnt from completed nanotoxicology studies: potential solutions to current and future challenges. Crit Rev Toxicol. 2013;43(1):1-20.

2. Panyam J, Labhasetwar V. Biodegradable nanoparticles for drug and gene delivery to cells and tissue. Adv Drug Deliv Rev. 2003;55(3): 329-347.

3. Gou M, Wei X, Men K, et al. PCL/PEG copolymeric nanoparticles: potential nanoplatforms for anticancer agent delivery. Curr Drug Targets. 2011;12(8):1131-1150.

4. Gou M, Shi H, Guo G, et al. Improving anticancer activity and reducing systemic toxicity of doxorubicin by self-assembled polymeric micelles. Nanotechnology. 2011;22(9):095102.

5. Wang BL, Shen YM, Zhang QW, et al. Codelivery of curcumin and doxorubicin by MPEG-PCL results in improved efficacy of systemically administered chemotherapy in mice with lung cancer. Int J Nanomedicine. 2013;8:3521-3531.

6. Lei N, Gong C, Qian Z, et al. Therapeutic application of injectable thermosensitive hydrogel in preventing local breast cancer recurrence and improving incision wound healing in a mouse model. Nanoscale. 2012;4(18):5686-5693.

7. Gong CY, Wu QJ, Dong PW, et al. Acute toxicity evaluation of biodegradable in situ gel-forming controlled drug delivery system based on thermosensitive PEG-PCL-PEG hydrogel. J Biomed Mater Res B Appl Biomater. 2009;91(1):26-36.

8. Zhao B, Wang XQ, Wang XY, et al. Nanotoxicity comparison of four amphiphilic polymeric micelles with similar hydrophilic or hydrophobic structure. Part Fibre Toxicol. 2013;10:47.

9. Pope CA 3rd, Burnett RT, Thurston GD, et al. Cardiovascular mortality and long-term exposure to particulate air pollution: epidemiological evidence of general pathophysiological pathways of disease. Circulation. 2004;109(1):71-77.

10. Brook RD, Rajagopalan S, Pope CA 3rd, et al; American Heart Association Council on Epidemiology and Prevention, Council on the Kidney in Cardiovascular Disease, and Council on Nutrition, Physical Activity and Metabolism. Particulate matter air pollution and cardiovascular disease: an update to the scientific statement from the American Heart Association. Circulation. 2010;121(21):2331-2378.

11. Crouse DL, Peters PA, van Donkelaar A, et al. Risk of nonaccidental and cardiovascular mortality in relation to long-term exposure to low concentrations of fine particulate matter: a Canadian national-level cohort study. Environ Health Perspect. 2012;120(5):708-714.

12. Nelin TD, Joseph AM, Gorr MW, Wold LE. Direct and indirect effects of particulate matter on the cardiovascular system. Toxicol Lett. 2012;208(3):293-299.

13. Karottki DG, Beko G, Clausen G, et al. Cardiovascular and lung function in relation to outdoor and indoor exposure to fine and ultrafine particulate matter in middle-aged subjects. Environ Int. 2014;73: 372-381. 
14. Weichenthal S, Villeneuve PJ, Burnett RT, et al. Long-term exposure to fine particulate matter: association with nonaccidental and cardiovascular mortality in the agricultural health study cohort. Environ Health Perspect. 2014;122(6):609-615.

15. Du Z, Zhao D, Jing L, et al. Cardiovascular toxicity of different sizes amorphous silica nanoparticles in rats after intratracheal instillation. Cardiovasc Toxicol. 2013;13(3):194-207.

16. Duan J, Yu Y, Li Y, Sun Z. Cardiovascular toxicity evaluation of silica nanoparticles in endothelial cells and zebrafish model. Biomaterials. 2013;34(23):5853-5862.

17. Chen Z, Wang Y, Zhuo L, et al. Effect of titanium dioxide nanoparticles on the cardiovascular system after oral administration. Toxicol Lett. 2015;239(2):123-130.

18. Simeonova PP, Erdely A. Engineered nanoparticle respiratory exposure and potential risks for cardiovascular toxicity: predictive tests and biomarkers. Inhal Toxicol. 2009;21(Suppl 1):68-73.

19. Lewis DR, Petersen LK, York AW, et al. Sugar-based amphiphilic nanoparticles arrest atherosclerosis in vivo. Proc Natl Acad Sci US A 2015;112(9):2693-2698.

20. Yamamoto Y, Nagasaki Y, Kato Y, Sugiyama Y, Kataoka K. Longcirculating poly(ethylene glycol)-poly(D,L-lactide) block copolymer micelles with modulated surface charge. J Control Release. 2001; 77(1-2):27-38.

21. Lupi M, Colombo C, Frapolli R, et al. A biodistribution study of PEGylated PCL-based nanoparticles in C57BL/6 mice bearing B16/F10 melanoma. Nanotechnology. 2014;25(33):335706.

22. Napierska D, Thomassen LC, Rabolli V, et al. Size-dependent cytotoxicity of monodisperse silica nanoparticles in human endothelial cells. Small. 2009;5(7):846-853.

23. Eilken HM, Adams RH. Dynamics of endothelial cell behavior in sprouting angiogenesis. Curr Opin Cell Biol. 2010;22(5):617-625.

24. Dimmeler S, Zeiher AM. Endothelial cell apoptosis in angiogenesis and vessel regression. Circ Res. 2000;87(6):434-439.

25. Scholz S, Fischer S, Gundel U, Kuster E, Luckenbach T, Voelker D. The zebrafish embryo model in environmental risk assessment - applications beyond acute toxicity testing. Environ Sci Pollut Res Int. 2008;15(5): 394-404.

26. Chakravarthy S, Sadagopan S, Nair A, Sukumaran SK. Zebrafish as an in vivo high-throughput model for genotoxicity. Zebrafish. 2014;11(2): 154-166.

27. Bakkers J. Zebrafish as a model to study cardiac development and human cardiac disease. Cardiovasc Res. 2011;91(2):279-288.

28. Shen Y, Leng M, Yu H, et al. Effect of amphiphilic PCL-PEG nanomicelles on HepG2 cell migration. Macromol Biosci. 2015;15(3): $372-384$

29. Wen H, Cao J, Wang L, Chen Y, Luo X. Self assembly of hydrophobic star-branched PCL and PCL-b-PEG amphiphilic copolymer. Acta Polym Sin. 2011;(3):240-245.
30. Zhang Z, Qu Q, Li J, Zhou S. The effect of the hydrophilic/hydrophobic ratio of polymeric micelles on their endocytosis pathways into cells. Macromol Biosci. 2013;13(6):789-798.

31. Thisse C, Thisse B. High-resolution in situ hybridization to wholemount zebrafish embryos. Nat Protoc. 2008;3(1):59-69.

32. Chakraborty A, Uechi T, Higa S, Torihara H, Kenmochi N. Loss of ribosomal protein L11 affects zebrafish embryonic development through a p53-dependent apoptotic response. PLoS One. 2009;4(1):e4152.

33. Miller GW, Labut EM, Lebold KM, Floeter A, Tanguay RL, Traber MG. Zebrafish (Danio rerio) fed vitamin E-deficient diets produce embryos with increased morphologic abnormalities and mortality. J Nutr Biochem. 2012;23(5):478-486.

34. Lee KJ, Nallathamby PD, Browning LM, Osgood CJ, Xu XH. In vivo imaging of transport and biocompatibility of single silver nanoparticles in early development of zebrafish embryos. ACS Nano. 2007; 1(2):133-143.

35. Osborne OJ, Johnston BD, Moger J, et al. Effects of particle size and coating on nanoscale $\mathrm{Ag}$ and $\mathrm{TiO}(2)$ exposure in zebrafish (Danio rerio) embryos. Nanotoxicology. 2013;7(8):1315-1324.

36. Zhao X, Wang S, Wu Y, You H, Lv L. Acute ZnO nanoparticles exposure induces developmental toxicity, oxidative stress and DNA damage in embryo-larval zebrafish. Aquat Toxicol. 2013;136-137:49-59.

37. Kim KT, Tanguay RL. The role of chorion on toxicity of silver nanoparticles in the embryonic zebrafish assay. Environ Health Toxicol. 2014 29:e2014021.

38. Fent K, Weisbrod CJ, Wirth-Heller A, Pieles U. Assessment of uptake and toxicity of fluorescent silica nanoparticles in zebrafish (Danio rerio) early life stages. Aquat Toxicol. 2010;100(2):218-228.

39. Carmeliet P. Angiogenesis in health and disease. Nat Med. 2003; 9(6):653-660.

40. Ferrara N, Gerber HP, LeCouter J. The biology of VEGF and its receptors. Nat Med. 2003;9(6):669-676.

41. Habeck H, Odenthal J, Walderich B, Maischein H, Schulte-Merker S; Tübingen 2000 screen consortium. Analysis of a zebrafish VEGF receptor mutant reveals specific disruption of angiogenesis. Curr Biol. 2002;12(16):1405-1412.

42. Covassin LD, Villefranc JA, Kacergis MC, Weinstein BM, Lawson ND. Distinct genetic interactions between multiple Vegf receptors are required for development of different blood vessel types in zebrafish. Proc Natl Acad Sci U S A. 2006;103(17):6554-6559.

43. Gerber HP, McMurtrey A, Kowalski J, et al. Vascular endothelial growth factor regulates endothelial cell survival through the phosphatidylinositol 3'-kinase/Akt signal transduction pathway. Requirement for Flk-1/KDR activation. J Biol Chem. 1998;273(46):30336-30343.

44. Choi JE, Kim S, Ahn JH, et al. Induction of oxidative stress and apoptosis by silver nanoparticles in the liver of adult zebrafish. Aquat Toxicol. 2010;100(2):151-159. 


\section{Supplementary materials}

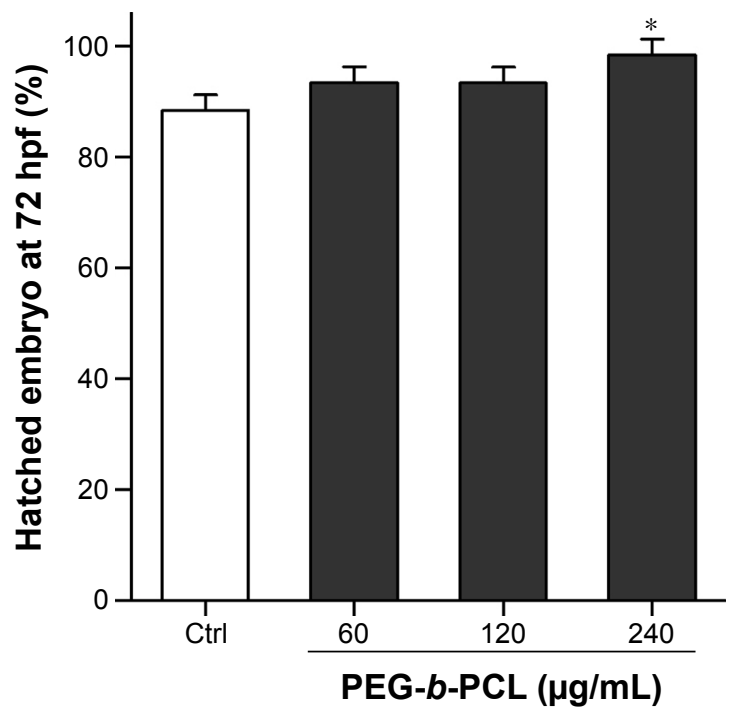

Figure SI Effect of PEG-b-PCL nano-micelle on embryo hatch.

Notes: Embryos were exposed to 0, 60, I20, and $240 \mu \mathrm{g} / \mathrm{mL}$ PEG-b-PCL nano-micelles. At $72 \mathrm{hpf}$, hatched embryos were counted. Data are expressed as mean \pm SEM from three independent experiments $(\mathrm{n}=60), * \mathrm{P}<0.05$.

Abbreviations: hpf, hours postfertilization; PEG-b-PCL, poly(ethylene glycol)-b-poly( $\varepsilon$-caprolactone); SEM, standard error of mean.

A

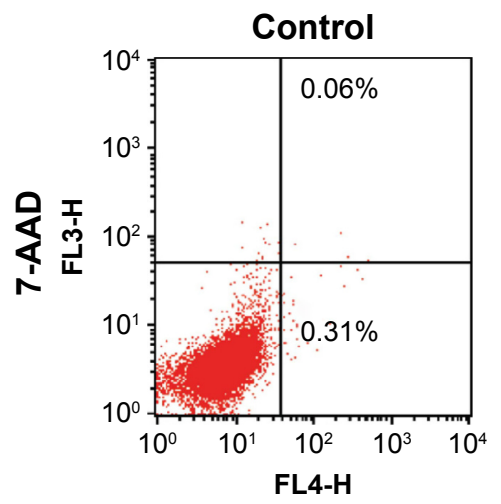

B

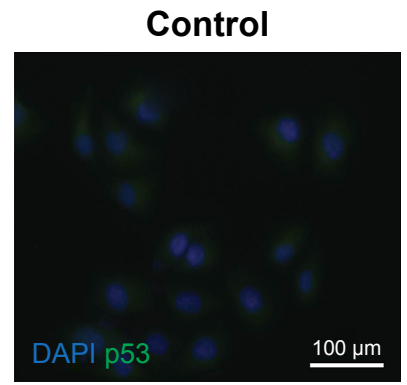

$30 \mu \mathrm{g} / \mathrm{mL}$ PEG-b-PCL nano-micelle

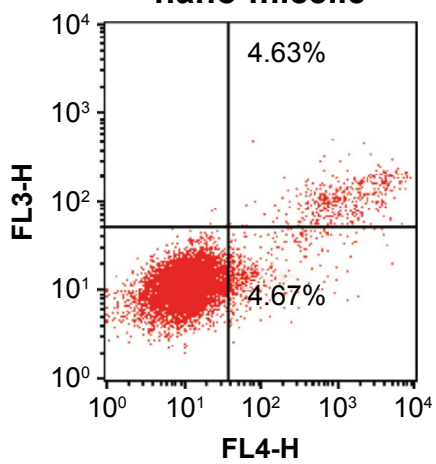

Annexin V-APC

$30 \mu \mathrm{g} / \mathrm{mL}$ PEG-b-PCL nano-micelle

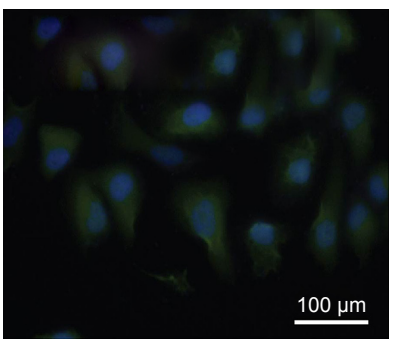

$60 \mu \mathrm{g} / \mathrm{mL}$ PEG-b-PCL nano-micelle

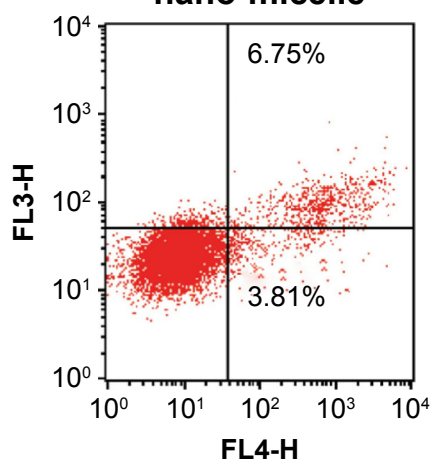

$60 \mu \mathrm{g} / \mathrm{mL}$ PEG- $b-P C L$ nano-micelle

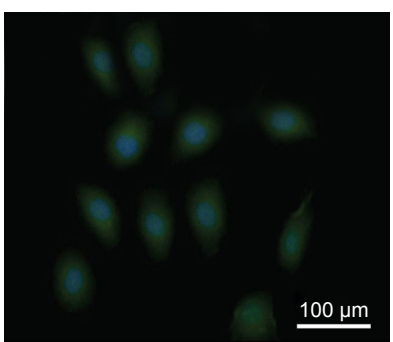

Figure S2 PEG-b-PCL nano-micelle induces apoptosis in endothelial cells in vitro.

Notes: (A) Apoptosis analysis of HUVECs by flow cytometry. HUVECs with or without PEG-b-PCL nano-micelles treatment were co-stained with APC-conjugated Annexin $V$ and 7-AAD. Cells positive for Annexin $V$ are in early apoptotic stage (lower right quadrant), and cells positive for Annexin V/7-AAD are in late apoptotic stage (upper right quadrant). (B) p53 immunostaining in HUVECs with or without PEG-b-PCL nano-micelles treatment. HUVECs were fixed with $4 \%$ PFA and co-stained with antibody against p53 (green) and 4',6-diamidino-2-phenylindole for DNA (blue).

Abbreviations: HUVECs, human umbilical vein endothelial cell; PEG-b-PCL, poly(ethylene glycol)-b-poly( $\varepsilon$-caprolactone); PFA, paraformaldehyde; AAD, aminoactinomycin D; APC, allophycocyanin. 
International Journal of Nanomedicine

Dovepress

\section{Publish your work in this journal}

The International Journal of Nanomedicine is an international, peerreviewed journal focusing on the application of nanotechnology in diagnostics, therapeutics, and drug delivery systems throughout the biomedical field. This journal is indexed on PubMed Central, MedLine, CAS, SciSearch ${ }^{\circledR}$, Current Contents ${ }^{\circledR} /$ Clinical Medicine,
Journal Citation Reports/Science Edition, EMBase, Scopus and the Elsevier Bibliographic databases. The manuscript management system is completely online and includes a very quick and fair peer-review system, which is all easy to use. Visit http://www.dovepress.com/ testimonials.php to read real quotes from published authors.

Submit your manuscript here: http://www.dovepress.com/international-journal-of-nanomedicine-journal 